\title{
Investigando a Dimensão Afetiva nos Mundos Virtuais 3D
}

Gleizer Bierhalz Voss, PPGIE/UFRGS, IFFar, gleizer.voss@iffarroupilha.edu.br Leandro Rosniak Tibola - PPGIE/UFRGS, URI, tibola@uri.edu.br

Manuel Constantino Zunguze - PPGIE/UFRGS, manuelczunguze@gmail.com Liane Margarida Rockenbach Tarouco - PPGIE/UFRGS, liane@penta.ufrgs.br Magda Bercht - PPGIE/UFRGS, bercht@inf.ufrgs.br

Resumo - Os mundos virtuais 3D têm sido utilizados para os mais diversos fins. Dentre estas atividades podem ser citadas as de lazer, comércio, relações sociais e educação. $\mathrm{O}$ uso de mundos virtuais para a educação tem ganhado cada vez mais atenção na medida em que o potencial destes ambientes é utilizado para pesquisas de ensino e de aprendizagem. Um dos aspectos ainda pouco explorado é de como e se os recursos dos mundos virtuais afetam os usuários, em especial a aparência e a adição de gestos aos avatares e aos personagens não-jogáveis. Este artigo tem como objetivo identificar como os agentes pedagógicos podem exercer uma influência afetiva positiva no sujeito (aluno) estimulando a aprendizagem.

Palavras-chave: mundos virtuais 3D; opensim; avatar; npc; afetividade.

\section{Investigating the Affective Dimension in 3D Virtual Worlds}

Abstract - 3D virtual worlds have been used for a variety of purposes. Among these activities can be mentioned those of leisure, commerce, social relations and education. The use of virtual worlds for education has gained increasing attention as the potential of these environments is used for teaching and learning research. One of the aspects still little explored is how and if the resources of the virtual worlds affect the users, especially with the appearance and gesture addition to avatars and non-player characters. This paper aims to identify how pedagogical agents can exert a positive affective influence on the subject (student) stimulating learning.

Keywords - 3D virtual worlds; opensim; avatar; npc; affectivity.

\section{INTRODUÇÃO}

A computação afetiva (CA) é uma subárea da inteligência artificial (IA) e um dos seus objetivos é melhorar a interação homem-computador (IHC) e a interação homem-robô (IHR) através da comunicação afetiva para criar uma interação mais intuitiva, envolvente e divertida (KARG et al., 2013). Para os autores, durante a interação, os estados afetivos podem ser expressos e reconhecidos através de expressões faciais, fala, movimentos corporais e parâmetros fisiológicos.

Além da aparência, a postura corporal e os gestos, servem como uma forma de comunicação e transmissão de mensagens. A linguagem corporal pode dizer muitas coisas para e sobre você. Então, os gestos possuem importância fundamental na comunicação humana, auxiliando no entendimento ou sendo a própria mensagem. Um exemplo básico são as repostas sim ou não para uma pergunta, que podem ser substituídas por um sinal do 
dedo polegar em riste e a mão fechada (confirmação) ou o dedo indicador apontando para cima com movimentos para direita e para esquerda com a palma da mão fechada (negação).

Outro ponto a ser destacado são os mundos virtuais 3D (MV3D), neste artigo tratados apenas como mundos virtuais (MV), onde os seres humanos podem ter a sensação de imersão em um ambiente tridimensional, por meio de seu avatar. Nos MV, os avatares podem interagir com objetos, com outros avatares e com personagens não-jogáveis (ou NPC, da sigla em inglês para Non-Player Characters), um tipo de avatar controlado pelo computador. Os avatares podem ser livremente definidos em seu gênero, forma física, vestimenta, cabelo, etc., em uma gama de itens personalizáveis. Isto permite ao usuário definir a aparência de seu avatar, de forma a representá-lo no MV. Como a comunicação nos MV é basicamente realizada por chat, a utilização de gestos pode incrementar o conteúdo das mensagens. Além disso, os avatares possuem um número prévio de gestos fornecidos por padrão pelo MV, porém eles devem ser "ativados" durante a conversa entre os avatares, o que demanda a associação prévia dos gestos ao avatar e sua "ativação" pelo usuário durante a conversa.

Neste sentido, percebe-se a possiblidade da utilização dos MV para fins educacionais. Um ponto de grande interesse nas pesquisas acadêmicas é a influência da afetividade na relação entre os participantes dos processos de ensino e aprendizagem, os quais nos MV são representados por avatares e NPC. Assim, o objetivo deste trabalho é investigar como e se os avatares e os NPC podem expressar emoções através de gestos nos MV.

O trabalho está dividido da seguinte forma: a seção 2 apresenta o referencial teórico, a seção 3 apresenta trabalhos relacionados, a seção 4 propõe possibilidades de expressão de afetividade nos usuários do OpenSim, especificamente através de aparência e gestos; por fim, na seção 5 são descritas as considerações finais e possibilidades de trabalhos futuros.

\section{FUNDAMENTAÇÃO TEÓRICA}

Esta seção descreve conceitos relativos à afetividade e aos MV, possibilitando o melhor entendimento da proposta do trabalho.

\subsection{Afetividade (Conceitos Básicos)}

É comum a sociedade ocidental seguir a visão dualista de Descartes sobre corpo e mente, porém, recentes pesquisas têm mostrado a importância da afetividade nas atividades cognitivas. Então, para entender como os alunos são afetados durante estas atividades, é necessário reconhecer os fenômenos afetivos.

Quanto ao conceito de afetividade, neste artigo, têm-se como base o conceito de Bercht (2001), como "o domínio das emoções propriamente ditas, dos sentimentos, das experiências sensíveis e, principalmente, da capacidade em se poder entrar em contato com sensações". De acordo com Longhi et al. (2009), "o estudo da afetividade é dificultado pela confusão de significados dos termos a ela relacionados".

Para Damásio (1996), a emoção é a percepção das alterações da paisagem do corpo e o sentimento é o processo de viver esta emoção, sendo que a essência de um sentimento é a percepção direta de uma paisagem específica: a paisagem do corpo. Tanto as emoções quanto os sentimentos dependem do sistema límbico, dos córtices pré-frontais do cérebro e 
de forma mais importante, dos setores cerebrais que recebem e integram os sinais enviados pelo corpo.

Já para Scherer (2000), emoção é um episódio relativamente breve de resposta sincronizada para a maioria ou todos os seres vivos, para a avaliação de um evento interno ou externo, como sendo de maior importância. São exemplos de emoções: alegria, medo, raiva, tristeza, desespero e vergonha.

Quando se passa a estudar o uso de uma figura que visa representar um humano dentro do sistema, torna-se inevitável considerar uma série de estudos em relação a como este personagem deve se comportar para parecer mais humano e, portanto, atingir seu principal objetivo. Com relação à sintetização de gestos, foram pesquisadas diversas maneiras de fazê-la para torná-la mais próxima da forma humana de se comportar (HARTMANN et al., 2005).

Neste sentido, Hartmann et al. (2005) propõe um modelo baseado em seis atributos que permite combinar seus atributos para avaliar a expressividade dos gestos. Os atributos são: (i) ativação global: tempo em que há movimentação durante um "turno" de conversação; (ii) extensão espacial: amplitude de movimentos; (iii) extensão temporal: duração dos movimentos; (iv) fluidez: suavidade e continuidade dos movimentos; (v) Força: propriedades dinâmicas do movimento; e (vi) Repetição: tendência de repetição rítmica de movimentos específicos.

Além de classificar os gestos, é importante perceber que eles podem completar a comunicação da linguagem verbal como uma linguagem não verbal. No que diz respeito a gestos e falas, existem três estratégias diferentes de distribuição, segundo Buisine e Martin (2007): redundante, complementar e controlada. Na primeira os gestos produzem informações duplicadas em relação à fala, na segunda a informação é distribuída entre gestos e fala e na terceira os gestos não possuem informação semântica e apenas a fala que passa as informações. Quando avaliada apenas a questão de fixação e entendimento das informações, a estratégia de redundância se mostrou mais apropriada.

No que tange aos gestos para um agente de software, Loyall e Bates (1997) reforçam o quanto a expressão de emoções pode influenciar na credibilidade da figura que visa representar um humano dentro do sistema. Jaques et al. (2012) apresentam uma proposta para dotar sistemas tutores inteligentes com habilidades sociais, justificando que esta abordagem é subsidiada, inclusive, por estudos da área de psicologia e pedagogia e que reforçam a ideia de que as emoções exercem influência direta na qualidade do aprendizado do aluno. Ainda conforme Jaques et al. (2012), a relação aluno professor também é marcada pela necessidade de um vínculo afetivo para que o aluno se sinta à vontade e motivado para aprender, e que as questões afetivas influenciam, inclusive nas escolhas das atividades que o aluno irá ou não realizar, bem como sua orientação frente a desafios.

\subsection{Mundos Virtuais 3D (MV)}

Os MV podem ser definidos como uma rede síncrona e persistente de pessoas, representadas como avatares, facilitada por computadores em rede (BELL, 2008). No contexto educacional os MV caracterizam-se por permitir explorar, testar e ampliar o entendimento de conceitos por meio da construção de artefatos significativos que podem ser compartilhados e personalizados pelos alunos. 
Como principais exemplos de plataformas de MV, têm-se o Second Life (uma plataforma proprietária, sendo uma das pioneiras e principal responsável pela popularização dos MV) e o OpenSimulator ou simplesmente OpenSim (um ambiente muito semelhante ao Second Life, porém com o diferencial de ser gratuito, open source e com uma vasta documentação no meio acadêmico). Para o desenvolvimento deste trabalho optou-se por utilizar a plataforma OpenSim, que além de ser um servidor de aplicações 3D de código aberto, é multiusuário e multiplataforma. Ele pode ser usado para criar MV, os quais podem ser acessados por uma variedade de clientes (viewers).

\subsubsection{Avatares e Agentes Artificiais}

Nos MV, os usuários são representados por seus avatares. A palavra avatar, segundo Schlemmer e Trein (2008) é o termo hindu utilizado para descrever uma manifestação corporal de um ser imortal, ou uma manifestação neste mundo de um ser pertencente a um mundo paralelo. Ainda para Mattar (2008) "você está situado, vê e ouve coisas daquele ponto de vista e então parece estar presente naquele ambiente".

Além dos avatares, outro elemento importante nos MV são os agentes artificiais, tratados neste artigo como NPC. Os NPC são personagens cuja suas ações são definidas através de conjuntos de programação compostos de roteiros (scripts) específicos e funções para executar determinadas atividades, ou seja, são personagens que não são interpretados por usuários reais, mas a partir de comandos predefinidos (HERPICH et al., 2016). Seu comportamento é geralmente reativo, desencadeado por certas ações ou diálogos com os avatares (Figura 1).

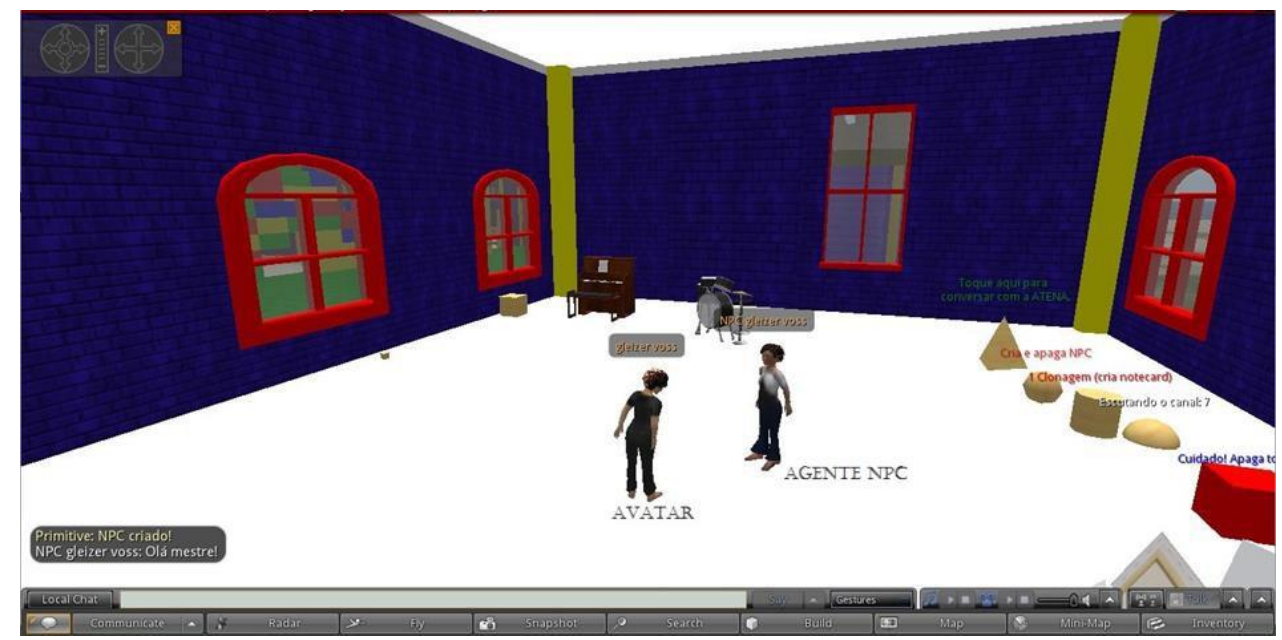

Figura 1: Interação entre usuário (avatar) e agente artificial (NPC) no MV

Fonte: Elaborado pelos autores

No OpenSim os NPC são criados utilizando as funções da OpenSimulator Scripting Language (OSSL), a qual é uma extensão da Linden Scripting Language (LSL) com instruções específicas para o servidor OpenSim (OPENSIM, 2017). A OSSL é composta por todas as funções LSL além de funções extras que iniciam com o prefixo "os" e podem ser escritas em um script da mesma forma que as funções "ll” da LSL.

Uma limitação apresentada pelos NPC em sua arquitetura e que parece ser recorrente em outras situações, é que seu modo padrão de comportamento está restrito a 
ações sequenciais e pré-definidas. Isto leva a algumas restrições em relação ao seu uso, tais como: baixa interatividade, incapacidade de adaptação a outro avatar, baixa capacidade de manipulação de objetos e baixa capacidade de atuação no ambiente, como seria esperado de um agente inteligente animado (APA), por exemplo. Conforme Motola e Jaques (2007), “os APA são agentes que tem um papel educacional ou pedagógico para facilitar ou aperfeiçoar a aprendizagem do aluno e que são representados por um personagem animado que interage com o aluno.

Neste sentido, Hodge et al. (2009) argumentam que os agentes são ideais para a modelagem de pessoas reais - eles são ativos e sociais, de maneira semelhante às pessoas reais. Além disso, os agentes podem ser usados para modelar os NPC, mantendo o controle de interesses individuais, motivações e objetivos nos mundos dos jogos. Porém, não há uma plataforma que possa ajudar os programadores a desenvolver e testar o comportamento dos agentes que precisam de autonomia, inteligência e interação com outros agentes e com o ambiente nos MV.

Uma vez que a programação das ações dos NPC é feita através de scripts prédeterminados, esta estratégia não permite muita liberdade para as ações dos agentes. Assim, percebe-se a necessidade de identificar formas de integrar as técnicas de IA que podem ser usadas nos MV.

\subsection{Inteligência Artificial em NPC}

Os NPC podem ser divididos em três categorias: inimigos, companheiros e personagens de apoio. Inimigos são os personagens que se opõem aos jogadores humanos no sentido pseudo-físico, atacando a força vital do jogador humano com armas ou magia. Os companheiros assumem o papel oposto e tentam proteger o jogador humano do qual eles são aliados. Alternativamente, personagens companheiros podem realizar tarefas que não sejam bélicas, tal como vender mercadorias em nome de seu aliado humano. Em alguns jogos, personagens parceiros podem ser ensinados por outros jogadores a realizar certos comportamentos. Consequentemente, o uso de agentes NPC, permite acrescentar um nível maior de interatividade, individualização de ações e suporte para a realização de atividades experimentais.

Conforme Herpich e Tarouco (2016), o uso dos NPC pode contribuir na construção do engajamento dos usuários, pois permitem focar a atenção dos usuários, indicando atividades e auxiliando-os na resolução de dúvidas sobre determinados tópicos. Uma abordagem comum para controlar o comportamento dos NPC é usar uma arquitetura de agente (SCHLEMMER e TREIN, 2008). A arquitetura do agente define um framework para os processos que um NPC deve executar repetidamente durante o jogo: capturar as variáveis do ambiente, raciocinar sobre os valores destas variáveis e agir no ambiente. Este conjunto de processos recorrentes é o loop de inteligência de um NPC.

Diferentes abordagens têm sido utilizadas para implementar o ciclo de inteligência. Conforme a abordagem escolhida, é determinada a complexidade, flexibilidade e adaptabilidade do comportamento do personagem (MATTAR, 2008). 


\section{TRABALHOS RELACIONADOS}

A aparência e a credibilidade dos agentes inteligentes, tais como os APA, têm sido tema de pesquisas, como as de Motola e Jaques (2007), Frozza et al. (2009), Boff e Oliveski (2012) e de Quarto et al. (2014). Estas pesquisas também refletem a necessidade de considerar a dimensão afetiva em ambientes virtuais de aprendizagem (AVA). Neste sentido, esta seção busca analisar e indicar alguns trabalhos a fim de estabelecer uma visão geral dessas questões nos AVA como um todo.

Alguns destes trabalhos propondo a utilização de agentes inteligentes ou Sistemas Tutores Inteligentes (STI) voltados para a área educacional foram considerados. Tais como Wenger, que ainda em 1987, escreveu um dos primeiros livros envolvendo sistemas tutores inteligentes (WENGER, 1987). Por sua vez, Bates (1994) escreveu sobre o papel da emoção em agentes acreditáveis (believable agents). Além desses, outros autores têm buscado avaliar e discutir questões relacionadas à melhoria da assistência ao aluno por meio da abordagem do componente afetivo na mediação da aprendizagem dos alunos, por exemplo: Bercht (2001), Jaques et al. (2012), entre outros.

Silva e Bernardi (2009) apresentam um APA chamado Cal, desenvolvido para interagir afetivamente com o aluno, de modo a facilitar a relação ensino-aprendizagem e também auxiliar o aluno na utilização do objeto de aprendizagem no qual o agente está inserido. Como as emoções que envolvem o aluno são consideradas impactantes no processo de ensino e aprendizagem, um dos objetivos das autoras com a inserção do agente pedagógico Cal foi de "minimizar a "distância" da comunicação entre o ambiente e o aluno, criando uma interação mais "amigável" e cordial."

Mais recentemente, Correia et al. (2014) descrevem a construção de um avatar 3D para interagir com os alunos em um AVA. Conforme os autores, o agente 3D pode expressar "de forma mais humanizada as emoções divergindo de outras formas de apresentação das emoções como técnicas de animação 2D e foto-sequência”. Já Scott et al. (2014) afirmam que os avatares podem ser empregados como uma ferramenta motivacional. Os autores contribuem para uma melhor compreensão de como criar e usar avatares como um meio para fornecer feedback aos alunos. Os resultados mostraram que os participantes consideraram os avatares visuais mais expressivos e mais fáceis de compreender do que suas contrapartes verbais. Constataram ainda que, os usuários respondem de forma diferente quando são apresentadas emoções negativas ou positivas. Os autores argumentam que este tipo de apresentação (mais visual) tem o potencial de envolver ou engajar os alunos por mais tempo, um fator fundamental para aplicações práticas (SCOTT et al., 2014).

\section{MANIFESTAÇÃO DE AFETIVIDADE ATRAVÉS DE GESTOS NO MV}

Uma vez que os comportamentos dos NPC são restritos a ações sequenciais e pré-definidas, existe a necessidade de ultrapassar as já mencionadas: baixa interatividade, baixa capacidade de manipulação de objetos e baixa ou nenhuma capacidade de atuação no ambiente, como seria esperado de um agente inteligente. Neste sentido, foram estudados os recursos disponíveis nos $\mathrm{MV}$, mais especificamente na plataforma OpenSim, que podem ser incorporados aos avatares e NPC. 
Dentre esses recursos estão os gestos. Eles estão pré-definidos na própria biblioteca do servidor, permitindo que sejam atribuídos tanto para avatares quanto NPC, a partir da definição da combinação de teclas para ativá-los. Um gesto pode ser formado por quatro elementos: animation, sound, chat e wait (animação, som, bate-papo e espera). Esses gestos podem ser ativados individualmente ou em conjunto e estão destacados nas Figuras 2 e 3.

Devido ao foco deste trabalho, a concentração será dada no elemento "animação" (animation). Na biblioteca do OpenSim estão disponíveis 73 animações diferentes. A partir dos conceitos de afetividade, emoções e sentimentos, apresentados anteriormente, foram destacadas 22 animações que melhor se enquadram nestes conceitos: Afraid (medo), Angry (raiva), Big Smile (sorrisão), Bored (entediado), Cry (choro), Disdain (desdém), Embarrased (embaraçado), Frown (sério), Impatient (impaciente), Jump for Joy (pular de alegria), Laugh (gargalhar), No (Unhappy) (não infeliz), No (não), Repulsed (repelido), Sad (triste), Shout (grito), Smile (sorriso), Surprise (surpresa), Tantrum (petulância), Worry (preocupação), Yes (Happy) (sim feliz) e Yes (sim). A partir desta seleção foram realizados experimentos, buscando atribuir animações ao avatar e o NPC, que permitissem ao usuário do MV perceber a manifestação de emoção através da animação.

A Figura 2 apresenta a manifestação de 4 dessas emoções. No quadro 1 da Figura 2 pode ser observado a animação "medo", no quadro 2 o "choro", no quadro 3 a "gargalhada" e no quadro 4 a "tristeza". Uma vez que se tratam de animações, não é possível demonstrar aqui toda a sequência dos movimentos através de uma imagem, porém, ela representa os movimentos do avatar em determinado momento, o que permite perceber que as representações destes gestos possuem um apelo visual que lembra a emoção propriamente dita.
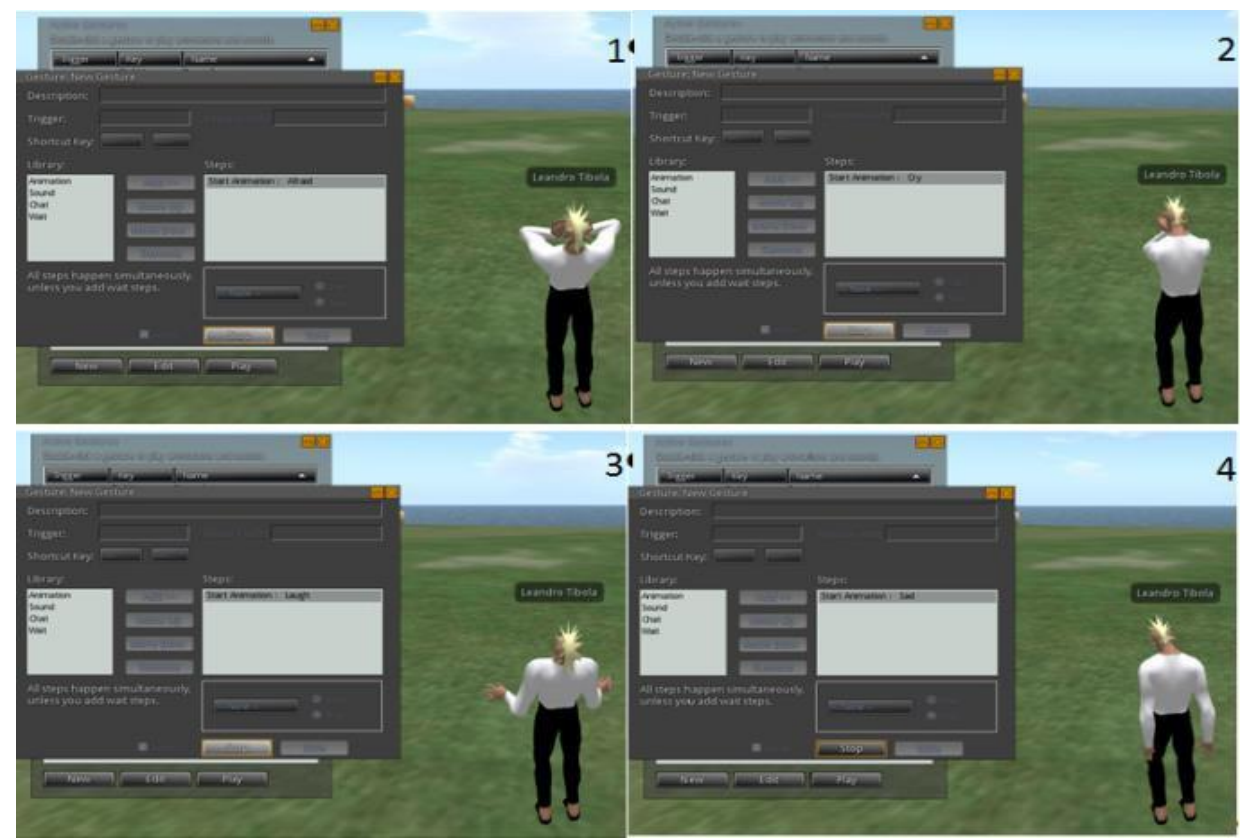

Figura 2: O avatar manifestando quatro emoções

Fonte: Elaborado pelos autores

Como descrito anteriormente, os gestos no OpenSim podem ser formados por 4 elementos. Quando utilizados em conjunto, sua efetividade tende a ser maior do que para o 
uso do elemento sozinho, pois há disponibilidade de informações por mais de um canal sensorial. Na Figura 3 é apresentado um avatar executando o gesto de "choro", sendo possível a visualização dos elementos animação (1) e do chat (3), adicionalmente, o elemento som (2) foi usado no experimento.

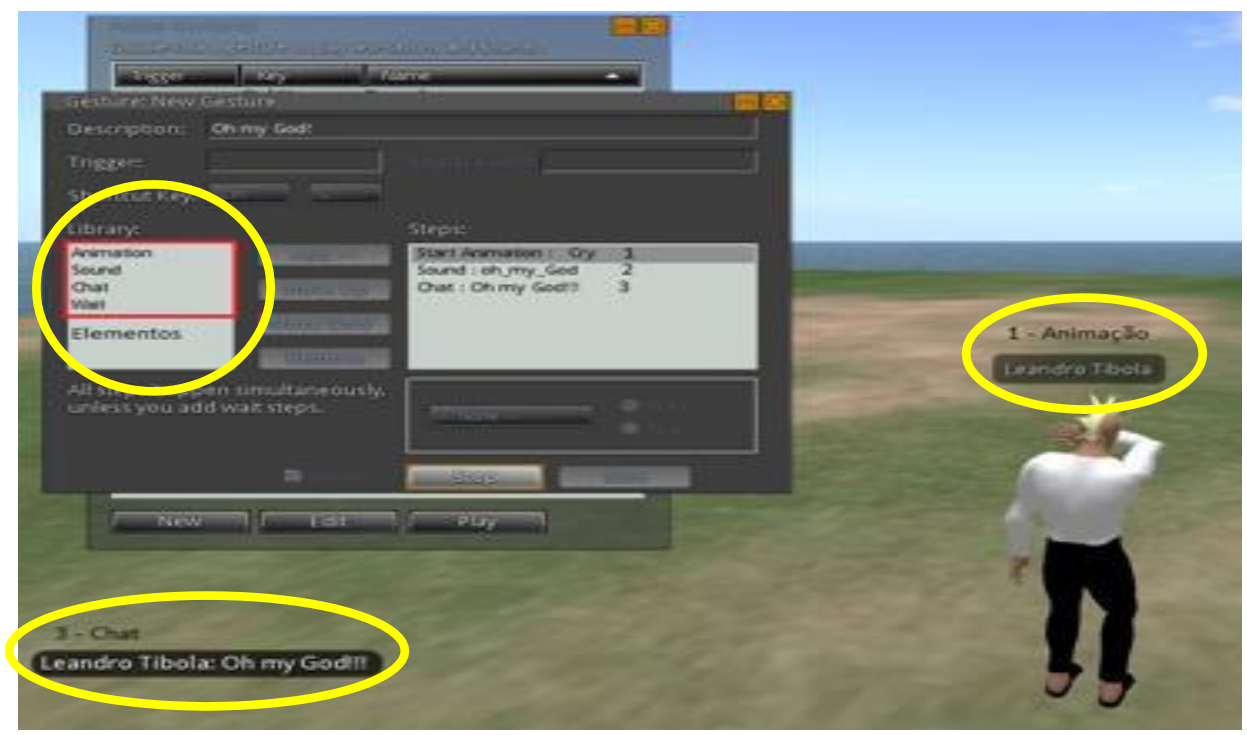

Figura 3: Elementos formadores de um gesto e a execução do gesto choro Fonte: Elaborado pelos autores

Uma questão necessária de ser pontuada é que alguns gestos não dispõem de visualização na perspectiva de visão apresentada nas Figuras 2 e 3, pois esta perspectiva é a do usuário que controla o avatar ou do NPC. Desta forma, alguns gestos são visíveis apenas para o outro avatar (interlocutor), o qual possui a visão frontal do avatar que está realizando as animações. Exemplos destes gestos são: Open Mouth, Disdain e Frown (boca aberta, desdém e rosto franzido).

\section{CONSIDERAÇÕES FINAIS}

A emoção pode ser entendida como a percepção das alterações da paisagem do corpo. Neste sentido, pode-se afirmar que um avatar ou um NPC, mesmo sem a forma física e sem o sistema límbico e os córtices pré-frontais de um cérebro (Damásio, 1996), possuem aparência e podem realizar gestos que manifestam em si uma emoção, a qual possui o potencial de afetar o usuário humano nos MV.

A criação e a utilização dos NPC estão diretamente relacionadas com a afetividade, uma vez que sua aparência carrega um potencial afetivo que pode desenvolver no usuário humano diversos fenômenos afetivos. Da mesma forma, os NPC podem ser dotados de gestos que manifestam emoções e também podem causar diferentes reações e sensações no usuário. Assim, percebe-se que a busca por dotar os NPC com dimensão afetiva não é algo novo, porém, não é muito usual e popular entre os desenvolvedores da área. Neste sentido, foram descritas algumas possibilidades e limitações da afetividade, dando exemplos do que tem sido feito e o que se pretende fazer com relação a este tema.

A aparência interfere na forma que o ser humano aceita ou rejeita o outro, uma ideia, um objeto, uma situação ou a si mesmo. Já a postura corporal e os gestos servem 
como uma forma de comunicação e transmissão de mensagens. Assim, aparência, postura corporal e gestos, quando associados a um NPC como agentes pedagógicos em MV, exercem uma grande influência afetiva no aluno estimulando a aprendizagem.

Por conseguinte, a principal contribuição do artigo foi investigar e constatar uma grande possibilidade de utilização de gestos, tanto em avatares quanto em NPC, a partir da capacidade dos mesmos de manifestar emoções através de movimentos, sons e textos. Os gestos estão disponíveis em uma biblioteca pré-definida do OpenSim e há a possibilidade de incrementá-los livremente. A utilização dos elementos em conjunto potencializa ainda mais a manifestação de emoções por parte dos avatares e dos NPC.

Como trabalhos futuros, pode-se apontar a possibilidade de identificar emoções através do chat entre o avatar do usuário humano e o NPC, permitindo que o NPC expresse os gestos correspondentes às emoções detectadas. Outro ponto de pesquisa é possibilitar ao usuário humano escolher a aparência do NPC, permitindo uma maior sinergia entre ambos. Por fim, cabe investigar quais são os ganhos em termos de desempenho na aprendizagem, comparando grupos que utilizam a expressão por gestos com grupos que não utilizam esta funcionalidade.

\section{REFERÊNCIAS}

BATES, J. The role of emotion in believable agents. Communications of the ACM, v. 37, n. 7, p. 122-125, 1994.

BELL, M. W. Toward a Definition of "Virtual Worlds". Journal for Virtual Worlds Research, v. 1, n. 1, 2008.

BERCHT, M. Em Direção a Agentes Pedagógicos com Dimensões Afetivas. UFRGS. Tese de Doutorado. 2001.

BOFF, E.; OLIVESKI, A. Personalização de Agentes Pedagógicos Animados. RENOTE, v. 10, n. 3, 2012.

BUISINE, S.; MARTIN, J. C. The effects of speech-gesture cooperation in animated agents' behavior in multimedia presentations. Interacting with Computers, v. 19, n. 4, p. 484-493, 2007.

CORREIA, C. L.; MONTANÉ, F. A. T.; TAMARIZ, A. R. Processo de criação e desenvolvimento de um agente pedagógico animado $3 \mathrm{~d}$ para ambientes virtuais de aprendizagem. InterScience Place, v. 1, n. 29, p 107-124, 2014.

DAMÁSIO, A. O Erro de Descartes. São Paulo: Companhia das Letras, 1996.

FROZZA, R.; KONZEN, A.; SILVA, D.; LUX, B.; KNIPHOFF, M. E. J.; BORIN, M. Dóris 3D: Agente Pedagógico baseado em Emoções. In: Anais do XX Simpósio Brasileiro de Informática na Educação. 2009.

HARTMANN, B.; MANCINI, M.; PELACHAUD, C. Implementing Expressive Gesture Synthesis for Embodied Conversational Agents. In: International Gesture Workshop. Springer, Berlin, Heidelberg, p. 188-199. 2005.

HERPICH, F.; NUNES, F. B.; VOSS, G. B.; MEDINA, R. D. Three-Dimensional Virtual Environment and NPC: A Perspective about Intelligent Agents Ubiquitous. In: Neto, Souza 
and Gomes (Orgs.); Handbook of Research on 3-D Virtual Environments and Hypermedia for Ubiquitous Learning. Hershey PA: IGI Global, p.510-536, 2016.

HERPICH, F.; TAROUCO, L. M. R. Engajamento de usuários em mundos virtuais: uma análise teórica-prática. RENOTE, v. 14, n. 1, 2016

HODGE, E.; COLLINS, S.; GIORDANO T. The virtual worlds handbook: how to use second life and other 3D virtual environments. Jones \& Bartlett Learning, 2009.

JAQUES, P. A.; NUNES, M. A. S.; ISOTANI, S.; BITTENCOURT, I. Computação afetiva aplicada a educação: dotando sistemas tutores inteligentes de habilidades sociais. In: Anais do Workshop de Desafios da Computação Aplicada à Educação, p. 50-59, 2012.

KARG, M.; SAMADANI, A; GORBET, R.; KUHNLENZ, K.; HOEY, J.; KULIC, D. Body Movements for Affective Expression: A Survey of Automatic Recognition and Generation. IEEE Transactions on Affective Computing, 4(4), 341-359, 2013.

LONGHI, M. T.; BEHAR, P. A.; BERCHT, M. A busca pela dimensão afetiva em ambientes virtuais de aprendizagem. In: Behar, P. (Orgs.). Modelos pedagógicos de educação à distância. Porto Alegre: Armed, 204-231, 2009.

LOYALL, A. B.; BATES, J. Personality-rich believable agents that use language. In: Proceedings of the first international conference on Autonomous agents. ACM, p. 106-113, 1997.

MATTAR, J. O Uso do Second Life como Ambiente Virtual de Aprendizagem. Fonte, Belo Horizonte, ano 5, n. 8, p. 88-95, 2008.

MOTOLA, R.; JAQUES, P. A. Uma Arquitetura Independente de Domínio e Plataforma para Apresentação de Comportamentos em Agentes Pedagógicos Animados. RENOTE, v. 5, n. 1, 2007.

OPENSIM. Página oficial da ferramenta. Disponível em: http://opensimulator.org/. Acesso em: abril, 2017.

QUARTO, C. C.; OLIVEIRA, A. N.; BERCHT, M. Agentes pedagógicos com dimensões afetivas: uma tecnologia no apoio ao ensino e a aprendizagem. In: Anais do XXV Simpósio Brasileiro de Informática na Educação. v. 25, p. 40, 2014.

SCHERER, K. Psychological models of emotion. In: BOROD, J. (Ed.). The neuropsychology of emotion. Oxford University Press, p.137-162. 2000.

SCHLEMMER, E.; TREIN, D. Criação de Identidades Digitais Virtuais para Interação em Mundos Digitais Virtuais em 3D. In: Congresso Internacional de EaD - ABED. 2008.

SCOTT, M.; PEREIRA, L.; OAKLEY, I. Show me or tell me: Designing avatars for feedback. Interacting with Computers, v. 27, n. 4, p. 458-469, 2014.

SILVA. T. G.; BERNARDI, G. Cal: um Agente Pedagógico Animado para Apoio em um Objeto de Aprendizagem para o Ensino de Matemática. In: Anais do XX Simpósio Brasileiro de Informática na Educação. 2009.

WENGER, E. Artificial intelligence and tutoring systems: computational and cognitive approaches to the communication of knowledge. Morgan Kaufmann, 1987. 